\title{
LA PROMOCIÓN DE LA VIRTUD CÍVICA MEDIANTE MECANISMOS DE DISEÑO INSTITUCIONAL
}

\section{THE PROMOTION OF CIVIL VIRTUE THROUGH INSTITUTIONAL DESIGN MECHANISMS}

\author{
Jordi Tena Sánchez ${ }^{1}$ \\ Universitat Autònoma de Barcelona
}

Recibido: 19-2-2015

Aceptado: 17-6-2015

\begin{abstract}
Resumen: El presente trabajo discute las posibilidades del diseño institucional como herramienta para la generación y extensión social de la virtud cívica. Se sostendrá que nuestros conocimientos acerca de cómo promover la virtud cívica son aún demasiado precarios como para plantearse ir más allá de algunas recomendaciones generales. En este sentido, probablemente la mejor estrategia consista en diseñar instituciones que economicen motivación y que promuevan el cumplimiento y la cooperación de cualquier tipo de agente, sea cual sea su motivación, y que empleen la virtud cívica existente sin erosionarla.
\end{abstract}

Palabras clave: mecanismos sociales; normas sociales; motivación; disposiciones de conducta.

\begin{abstract}
This paper explores institutional design strategies which could promote civic virtue. It will be argued that we do not have knowledge enough to go beyond of some general principles. In this sense, probably the best option is designing institutions which economize motivation and do not erode existing civic virtue.
\end{abstract}

Key words: social mechanisms; social norms; motivation; dispositions.

[1] (Jordi.Tena@uab.cat) Profesor del Departament de Sociologia de la Universitat Autònoma de Barcelona. Sus últimas publicaciones han sido: (2016): «Existe una Función «Informativa» de la Ley? Dos tests experimentales», Aposta. Revista de Ciencias Sociales, 68: 9-32.(2015): «Estrategias de Diseño Institucional y Cumplimiento. El Caso de las Nuevas Políticas de Seguridad Vial Aplicadas en España", Sortuz: Oñati Journal of Emergent Socio-Legal Studies, 7 (2), 1-19. 


\section{Introducción}

Con más de 20 siglos a sus espaldas, el de virtud cívica es uno de los conceptos más recurrentes de la historia de la filosofía y las ciencias sociales. Dicha recurrencia parece merecida dado que tradicionalmente se ha sostenido que la virtud cívica resulta imprescindible para la consecución de multitud de bienes sociales de primera magnitud ${ }^{2}$.

No obstante, llama la atención que pese a su relevancia teórica y política, la cuestión de cómo promover la virtud cívica a nivel social permanece mal comprendida. El objetivo del presente trabajo consiste, precisamente, en explorar algunas estrategias de diseño de instituciones formales (tales como leyes, políticas, regulaciones, ordenanzas, etc.) que bien podrían contribuir a la generación y extensión social de la virtud cívica. ${ }^{3}$

En este trabajo se adoptará una definición deliberadamente vaga de lo que debemos entender por virtud cívica que permita dar cabida a distintas concepciones del concepto. Se entenderá que lo que constituye la virtud cívica son cosas tales como motivaciones, disposiciones de conducta, valores o normas sociales públicamente orientadas, esto es, orientadas a la promoción del bien de la sociedad. ${ }^{4}$

Siguiendo libremente a Pettit (1999) el trabajo se organiza en base a dos grandes apartados: la promoción de la virtud cívica en contextos en los que esta es muy escasa (contextos de vicio) y su promoción en contextos en que ésta se encuentra ya ampliamente difundida (contextos de civilidad ampliamente difundida). Se concluirá que, pese a suponer uno de los principales retos a los que se enfrentan las democracias contemporáneas, nuestros conocimientos acerca de cómo promover la virtud cívica son aún demasiado precarios como para plantearse ir más allá de algunas recomendaciones generales.

\section{La promoción de la virtud en contextos de vicio}

El objetivo de promover la virtud cívica se concretará de modo distinto en función del contexto en el que nos encontremos. Podemos hallarnos, en primer lugar, en un contexto en el que la virtud cívica no se encuentre socialmente extendida, en el que no se dé una civilidad ampliamente difundida. Un contexto así puede aún concretarse, como mínimo, en tres escenarios diferenciables.

[2] Por ejemplo, véase Pettit (1999) para una discusión del papel de la virtud en la consecución de la libertad republicana.

[3] Se deja, por tanto, de lado el papel de otras herramientas (como la pedagogía o la propaganda). En este sentido, el trabajo entronca con la tradición republicana que, como es bien sabido, concibe al diseño institucional como la herramienta primordial para la promoción del cumplimiento y la civilidad (véase Pettit, 1999, p. 328).

[4] Véase Tena-Sánchez (2010) para una discusión detallada sobre la definición del concepto.

Thémata. Revista de Filosofía No53 (2016) pp.: 107-118. 
En primer lugar, puede que simplemente la gente no coopere en la promoción del bien de la sociedad, que se comporten como gorrones. Piénsese, por ejemplo, en un contexto de corrupción generalizada en el que los ciudadanos no valoran el bien común, no cumplen la ley, no pagan sus impuestos, etc. Este tipo de escenarios, empero, no serán por desgracia ni los más nocivos para el bien de la sociedad ni probablemente los casos más típicos de sociedad en la que la virtud cívica se halla ausente. Podemos encontrarnos también ante escenarios de corrupción generalizada en los que sí que existen, no obstante, normas de cooperación que son además cumplidas por la mayoría de los ciudadanos en la mayoría de las ocasiones. Un ejemplo de este tipo de contextos vendría dado por aquellos lugares en los que las mafias y las organizaciones criminales imponen su orden con un grado de conformidad relativamente alto entre la población.Un tercer caso posible de sociedad en la que no se da una civilidad ampliamente es aquel en que los ciudadanos cumplen la ley por voluntad propia, pero dicha ley no se encuentra orientada hacia el bien de la sociedad sino todo lo contrario. Un ejemplo extremo de este caso vendría dado por el apoyo popular a determinadas dictaduras. ${ }^{5}$ En una situación así, de hecho, lo que la virtud requeriría de los ciudadanos sería que se opusiesen activamente a la ley o, en su caso, al régimen en su conjunto. ${ }^{6}$

Esta clasificación de tres casos pretende ser meramente ilustrativa de algunos de los muchos tipos de situaciones comunes en los que no se da una civilidad ampliamente difundida. Obviamente, no es ni trata de ser exhaustiva y, además, los casos citados tampoco son mutuamente excluyentes.

En contextos de este tipo el objetivo debe ser la creación de virtud cívica ex novo ${ }^{7}$. A partir de la definición laxa de virtud cívica que se ha ofrecido en la introducción, el objetivo de crear virtud cívica aún podría diseccionarse en algunos objetivos diferentes, a saber: crear determinadas motivaciones y disposiciones de conducta (como el altruismo, la solidaridad o la generosidad)

[5] Véase, por ejemplo, Kuran (1995) para un análisis del apoyo popular a las dictaduras de la Europa del Este.

[6] No pretende sostenerse que las dictaduras constituyan los únicos tipos de regímenes en los que no se promueve el bien de la sociedad o en los que se promueve el mal de la misma. Obviamente, en las democracias liberales no se promueve el bien todo lo que sería posible y, en muchas ocasiones, se promueve el mal. En este sentido, el bien de la sociedad constituye un ideal hacia el que orientar las políticas y no tanto un objetivo político factible y fácilmente alcanzable. También, claro está, en las democracias liberales se dictan frecuentemente leyes injustas y también en esos casos la virtud puede requerir a los ciudadanos que se opongan a las mismas.

[7] En el tercer caso, el objetivo debería ser también (e incluso previamente), claro está, derribar el régimen en cuestión. Se dejan de lado aquí estas complicaciones.

Thémata. Revista de Filosofía №53 (2016) pp.: 107-118. 
y crear normas sociales públicamente orientadas (por ejemplo, normas que prescriban la cooperación en la promoción del bien de la sociedad).

Por lo que se refiere al primer objetivo, el de promover determinados tipos de motivaciones como el altruismo o la generosidad, ni tan sólo podemos estar seguros de que tenga demasiado sentido planteárselo. Los tipos de motivaciones humanas son universales y, muy probablemente, innatas. ${ }^{8}$

No obstante, evidentemente hay personas que tienen una mayor propensión (una disposición de conducta) a actuar motivadas por un determinado tipo de motivaciones y otras que la tienen para actuar motivadas por otras. Es indudable, por ejemplo, que existen personas más altruistas que otras, o, como mínimo, con mayor tendencia a actuar de forma altruista en un determinado tipo de situaciones. Pero, ¿cómo se pueden promover socialmente estas disposiciones? En primer lugar, no es ni mucho menos descartable que tengan, al menos parcialmente, un origen biológico. Por otro lado, suele sostenerse, y es muy plausible suponer que realmente sea así, que instrumentos como la educación desempeñan un importante papel en este punto. No obstante, desconocemos demasiado el funcionamiento de estos procesos como para plantearnos un programa sistemático de intervención en este sentido que vaya más allá de algunos principios genéricos, como sostener que es importante fomentar que los niños aprendan a compartir.

Finalmente, por lo que respecta a la cuestión de cómo hacer emerger normas sociales públicamente orientadas (y debilitar las normas de vicio), el resultado tampoco es demasiado alentador. Desconocemos en una importante medida los procesos que llevan a la emergencia o desaparición de una norma determinada. Tal y como argumenta Jon Elster (2007: 358-360), existen algunas normas que son socialmente útiles en el sentido fuerte de que hacen que todo el mundo esté mejor. Normas como, por ejemplo, las que proscriben escupir en el autobús u orinar en la vía pública generan bienes públicos en el sentido de que promueven externalidades positivas o evitan negativas. Parece intuitivamente plausible suponer que esas normas existen precisamente porque promueven dichos bienes, pero el problema radica en identificar el mecanismo concreto a través del cual pueden haber emergido. Generalmente, las normas que regulan externalidades existen en contextos en los que también existe una ley que regula la misma conducta. Éste es, sin ir más lejos, el caso de los ejemplos a los que acaba de hacerse referencia: escupir u orinar en la vía pública, no solo está mal visto, sino que está prohibido por la ley. Más aún, en no pocas ocasiones, primero la administración aprueba una ley que regula una externalidad y posteriormente emerge una norma que prescribe o proscribe la misma conducta que la ley y que refuerza a ésta última. En cambio, no está tan claro que ese tipo de normas emerjan habitualmente sin la existencia previa

[8] Véase Tena-Sánchez (2010b) para una discusión.

Thémata. Revista de Filosofía No53 (2016) pp.: 107-118. 
de una ley. Una vez que la norma existe, generalmente ésta se mantiene aun después de que la ley sea derogada. Estos argumentos irían a favor de la tesis defendida en este trabajo según la cual el diseño institucional puede jugar un papel central en la promoción de la virtud cívica. No obstante, el problema sigue siendo identificar el mecanismo o mecanismos que explican el modo en que la ley causa la emergencia de normas. ${ }^{9}$

\section{La promoción de la virtud cívica en contextos de civilidad ampliamente difundida}

\subsection{Motivar a los villanos}

Una cuestión diferente de las anteriores tiene que ver con el significado de promover la virtud cívica en contextos donde ésta ya se da en un grado razonable, donde existe ya una civilidad ampliamente difundida, donde la gente ya coopera por voluntad propia de forma más o menos habitual en la promoción del bien de la sociedad. Una vez más, este problema puede ser diseccionado en algunos otros problemas más concretos. En primer lugar, incluso en un contexto en el que la virtud cívica se halle ampliamente difundida siempre encontraremos potenciales villanos. Siempre habrá ciudadanos que no se hallen motivados por el bien de la sociedad y que, en cambio, traten de promover su propio interés particular a costa del bien común en cuanto tengan la posibilidad. ${ }^{10}$ Sin necesidad de llegar hasta casos extremos, la mayoría de los ciudadanos, pese a ser generalmente virtuosos, pueden actuar como villanos en determinadas circunstancias. Una primera pregunta pertinente puede ser, por tanto, ¿cómo cambiar la motivación de estos ciudadanos? Nótese que en este caso es especialmente importante distinguir entre conseguir que el agente se limite a cumplir merced a las sanciones positivas y/o negativas derivadas del cumplimiento y/o el incumplimiento, y conseguir que cambie su motivación y pase a cumplir por voluntad propia (que se convierta en un virtuoso). ${ }^{11}$

El problema de la motivación de los potenciales villanos vuelve a enfrentarnos al problema de cómo crear o cambiar las motivaciones de los indi-

[9] Véase el citado Elster (2007, cap. 22) para una discusión más detallada de los argumentos que acaban de ofrecerse.

[10] La discusión conecta aquí con los debates en el ámbito de criminología acerca de cómo combatir la criminalidad y los diferentes tipos de justicia (restaurativa, disuasoria eincapacitatoria). No obstante, se dejan aquí de lado estas cuestiones específicas.

[11] Otro caso particular de este problema que también se dejará de lado en estas páginas tiene que ver con cómo conseguir que los nuevos ciudadanos, los niños, interioricen las disposiciones y motivaciones adecuadas. Abordar dicha cuestión supondría entrar en debates muy específicos y complejos relacionados con la educación y la pedagogía. Además, nuevamente,

Thémata. Revista de Filosofía $\mathrm{N}^{\circ} 53$ (2016) pp.: 107-118. 
viduos. Nuevamente, sabemos poco acerca de los mecanismos concretos que podrían producir este resultado y de cómo favorecer su actuación. No obstante, merece la pena traer aquí a colación algunos mecanismos identificados por la literatura y que tal vez pudiesen ser activados con el correcto diseño institucional. La lista no pretende ser exhaustiva.

El primero de ellos es el conocido mecanismo de las preferencias adaptativas o de uvas amargas ${ }^{12}$. Dicho mecanismo consiste en una forma irracional de cambio o formación de preferencias. De forma general, se desencadena como respuesta al malestar psíquico (disonancia cognitiva) derivado de la frustración de un deseo. Un agente que alberga un deseo que no puede satisfacer se sentirá frustrado por ello. Siendo esto así, es posible que sus deseos cambien y se adapten a su conjunto de oportunidad disponible de modo que su malestar desaparezca. En lo que respecta a este trabajo, resulta plausible suponer que algunos individuos que querrían actuar de forma viciosa pero que no pueden hacerlo debido a que se encuentran inmersos en un entramado social e institucional en el que su conducta sería descubierta y sancionada, acaben por desear de buena fe cumplir con lo que establecen las reglas de cooperación. Este podría ser el caso, por ejemplo, de un trabajador autónomo (un mecánico, un electricista, etc.), que preferiría no cargar el IVA a sus clientes pero que vive en un país nórdico donde existen fuertes normas sociales que proscriben este tipo de prácticas así como importantes mecanismos de control del fraude.

Un segundo mecanismo similar al anterior es la transmutación de preferencias. Imaginemos que un ciudadano se encuentra en una situación susceptible de requerir que falsifique alguna de sus preferencias privadas. Imaginemos, por ejemplo, el caso de un regidor de un pequeño Ayuntamiento que desea que se apruebe algún tipo de reglamento que lo beneficia a él y que trata de defender la medida en el pleno municipal. En un contexto deliberativo como ese, dicho regidor no puede argumentar que habría que hacer $\mathrm{X}$ porque de esa manera sus intereses resultarán favorecidos en detrimento de los de los demás vecinos. De este modo, en primer lugar, puede tergiversar sus preferencias de forma consciente y voluntaria. Puede disfrazar su interés egoísta vistiéndolo con la retórica del bien común. Podría argumentar, por ejemplo, que lo mejor para el bien común del municipio es hacer X. Pero es probable que el citado regidor no se limite a tergiversar sus preferencias sino que acabe por transmutarlas. Verse obligado a ocultar sus auténticas motivaciones y a

sabemos demasiado poco acerca del funcionamiento de estos procesos como para plantearnos un programa sistemático de intervención en este sentido que vaya más allá de algunas ideas y principios vagos como que un hogar estructurado con unos padres que dediquen tiempo y amor a sus hijos será mejor para el desarrollo de estos que uno desestructurado o con unos padres irresponsables.

[12] La discusión de éste y los siguientes mecanismos se basa en Elster (1988, 1990, 1995, 2002 y 2007).

Thémata. Revista de Filosofía No53 (2016) pp.: 107-118. 
actuar cínicamente puede generarle de nuevodisonancia cognitiva. Es posible que empiece a pensar que es un hipócrita y un villano que trata de aprovecharse de la buena fe de sus conciudadanos. Sometido a una tensión psíquica de esa naturaleza, no resulta improbable que sus preferencias se transmuten y acabe interiorizando los argumentos que sostiene públicamente.

El conformismo y la conformidad constituyen otros dos mecanismos que podrían operar en estos contextos. Un individuo es conformista cuando adapta sus deseos a los de otros o a la inercia común. La conformidad es diferente del conformismo. La conformidad es un deseo causado por un impulso a ser como los demás. Dicho mecanismo implica que la conducta de otros se incorpora a la causación de los deseos de un agente, el conformismo implica que esas personas se incorporan a la descripción del objeto de deseo del agente. La conformidad puede producir conformismo pero también anticonformismo. Nuevamente, es fácil ver como algunos de estos mecanismos pueden llevar a un agente a adoptar los deseos requeridos por el bien común. Por ejemplo, si alguien es conformista y se halla en un contexto de civilidad ampliamente difundida, tenderá a asumir como propios los deseos de las personas a quien quiera parecerse.

Otro mecanismo que puede resultar de utilidad en la presente discusión es el que Bicchieri (2006) denomina la falacia naturalista. Como es bien sabido, derivar eldebe del es constituye un error lógico. Los seres humanos incurrimos empero frecuentemente en dicho error. En este sentido, un ciudadano que vive en una sociedad en la que las instituciones promueven generalmente el bien de la misma puede fácilmente asumir que es natural que esto sea así y que así debería ser siempre.

T. Kuran (1995) hace referencia a algunos mecanismos más que pueden ser también de aplicación en este caso. El primero de ellos es la heurística de la prueba social. La racionalidad humana, como es sabido, es limitada. Los seres humanos tan sólo podemos albergar un conocimiento experto sobre un pequeño número de temas (quizá sobre ninguno) pero, no obstante, eso no impide que nos formemos creencias sobre un gran número de cuestiones (North 2005). Una de las heurísticas que utilizamos para formarnos creencias sobre materias en las que no tenemos la posibilidad (ni, frecuentemente, la voluntad) de recopilar más información es la heurística de la prueba social. Si existe un amplio consenso en torno a una determinada cuestión es probable que tendamos a asumir como válida la opinión de la mayoría. De este modo, es probable esperar que muchos ciudadanos que vivan en un contexto de civilidad ampliamente difundida terminen por aceptar la bondad de dicho contexto, aun cuando en un primer momento hubiesen sido reacios al mismo.

Un segundo mecanismo mencionado por Kuran que podría tener exactamente el mismo efecto es la repetición. La repetición de un mismo mensaje 
hace que los individuos se familiaricen con el mismo y le otorguen mayor veracidad.

Kuran $(1995,1998)$ distingue también entre lo impensable y lo impensado. Algunas de nuestras preferencias pueden situarse en el terreno de lo impensable si existen poderosas presiones sociales o legales que nos impiden expresarlas. Por su parte, una opción entra en el terreno de lo impensado cuando ni tan sólo podemos llegar a planteárnosla dentro de nuestro conjunto de opciones posibles. Si las presiones sociales existentes impiden que los individuos que albergan un determinado tipo de preferencias puedan expresarlas, dichas preferencias tenderán a convertirse en (o permanecer) impensadas para los demás. Paulatinamente, el conjunto de los individuos prestará cada vez menos atención a las posibilidades de dichas opciones,mientras que se centrará en los beneficios posibles de las opciones disponibles. La heurística de la prueba social trabajará decisivamente en esta dirección. En el largo plazo, las opciones apartadas de la esfera de la discusión pública tenderán a volverse impensadas para las nuevas generaciones. Los individuos que albergaban las preferencias impensables pueden acabar modificando también sus preferencias a lo largo de este proceso. Un primer mecanismo que puede operar aquí es el fundamental attribution error (Bicchieri 2006, Elster 2007, Kuran 1995). Los seres humanos tendemos a infravalorar la distancia entre nuestras preferencias públicas y privadas. Así, un individuo que reiteradamente se vea obligado a falsear una determinada preferenciapuede acabar pensando que, en realidad, la falsificación no es demasiado importante o, incluso, que la preferencia que expresa públicamente es, de hecho, su auténtica preferencia. Un segundo mecanismo que puede operar en el mismo sentido descansa sobre el poder de las creencias públicamente aceptadas. Cualquier creencia servirá para dar cuenta de unos hechos mientras que será discutida por otros. Las creencias públicamente aceptadas resultan ampliamente incuestionadas y no están obligadas a dar cuenta de sus debilidades, mientras que los individuos que alberguen creencias impensables se verán constantemente presionados para dar cuenta de las limitaciones de las mismas. Este hecho puede darse además en un contexto en el que el individuo no tenga acceso a información relevante ni esté en contacto con otros individuos que albergan sus mismas creencias. Estas presiones, unidas a las de la heurística de la prueba social, pueden dar lugar a un progresivo debilitamiento de las creencias en cuestión así como de las preferencias asociadas a las mismas (Kuran, 1995, 1998).

Pese a que es de esperar que los mecanismos mencionados por Kuran operen sobre todo en contextos donde la crítica y la libertad de expresión (como las dictaduras que él estudió), nada impide que puedan operar también en las democracias liberales, incluso en aquéllas en que se den mayores cuotas de civilidad. En una comunidad cívica es de esperar que exista un cuerpo bien

Thémata. Revista de Filosofía №53 (2016) pp.: 107-118. 
establecido de normas sociales orientadas hacia el bien común. Como es bien sabido, criticar una norma acarrea costes para quien la critica. De este modo, en un contexto de civilidad ampliamente difundida habrá individuos viciosos que se limiten a cumplir de forma hipócrita, falsificando sus preferencias, y que sean susceptibles de verse afectados por los mecanismos estudiados por Kuran.

\subsection{Definir una situación como del tipo en el que resulta perti- nente ser virtuoso}

El problema la promoción de la virtud en un contexto de civilidad ampliamente difundida tiene, como mínimo, una dimensión adicional a la que acaba de plantearse. En determinadas circunstancias los agentes pueden albergar disposiciones virtuosas y, sin embargo, abstenerse de cumplir con lo que el bien de la sociedad requeriría de ellos, simplemente porque no identifican dichas situaciones como del tipo en el que resulta pertinente cooperar en la promoción del bien común.

Una vez más, es necesario recordar en este punto que los seres humanos albergamos motivaciones plurales. Todos los seres humanos albergamos motivaciones como el altruismo o la solidaridad. No se trata, por tanto, de que haya personas que actúan siempre de forma altruista, otras que lo hacen de forma egoísta, otras motivadas por normas, etc. Se trata más bien de que todos seguimos normas en determinadas situaciones, somos altruistas en otras, egoístas en otras, etc. Una cuestión crucial en este sentido es, ¿en qué tipo de situaciones actuamos guiados por una motivación u otra y por qué esto es así?

En un ejercicio de lo que podríamos denominar como folk psychologyingenua, los seres humanos solemos concebirnos como guiados por motivaciones que tienen su origen en disposiciones y rasgos de carácter más o menos estables en el tiempo (Bicchieri, 2006). En este sentido, decimos de alguien que es honesto cuando consideramos que tiene interiorizada una norma de honestidad que guía su conducta habitualmente. En un extremo, siguiendo la postura de los clásicos al respecto, podríamos suponer que todas las virtudes van juntas de modo que quien alberga una las alberga todas y constituye una persona moralmente buena; a la inversa, lo mismo sucedería con los vicios (Elster, 2007).

Esta concepción de la conducta y la motivación humana ha sido dominante durante siglos, no ya en la psicología popular sino en la filosofía y en las ciencias sociales. No obstante, hace tiempo que desde la psicología cognitiva y otras ciencias aledañas se viene aportando evidencia empírica que parece mostrar que se trata de una concepción errónea en una importante medida, víctima del mencionado fundamental attribution error. Ser honesto es una característica personal pero, en un aspecto importante, es también una

Thémata. Revista de Filosofía №53 (2016) pp.: 107-118. 
característica local o situation-specific,en el sentido de que en muchas ocasiones el factor con mayor poder explicativo de la conducta es la situación y no la persona ${ }^{13}$. Elster (2007) cita el ejemplo de un experimento con estudiantes de teología en el que a unos se les pidió que discutiesen durante un rato sobre la parábola del buen samaritano y a otros sobre un tema sin trascendencia moral. Terminada la discusión se les pidió que se dirijan a otro lugar. A unos se les dijo que los estaban esperando y que debían darse prisa, al resto no se le dio ninguna instrucción especial. En el trayecto hacia el otro lugar encontraron a un indigente pidiendo limosna (uno de los experimentadores). Sólo el 10\% de los sujetos a los que se les indicó que debían darse prisa se detuvo a ayudar al indigente, en el otro grupo más de la mitad de los sujetos se detuvo. Ni el tema sobre el que habían estado discutiendo ni ningún otro factor similar controlado por los experimentadores pareció tener incidencia alguna en el resultado; por el contrario, parece que fue el hecho de encontrarse en una situación relajada o tener prisa el factor con mayor poder explicativo de la conducta.

De manera general, tal y como explicita Bicchieri (2006, cap. 2), los diferentes contextos en los que nos encontramos presentan unas características que resultan prominentes para nosotros, se trata de señales que captan nuestra atención (attentioncues). A través de toda una serie de procesos que funcionan por debajo de nuestra conciencia, los seres humanos interpretamos esas señales y categorizamos la situación como de un tipo u otro. Dependiendo de las señales que percibamos y de cómo las interpretemos podemos categorizar una situación como una del tipo en la que opera una norma de equidad o en la que lo pertinente es perseguir el propio interés, etc. Una vez que hemos categorizado una situación se activan nuestros schemata. Los schematason estructuras cognitivas que representan conocimiento almacenado sobre personas, eventos, roles, expectativas compartidas de lo que se espera que suceda, preferencias, etc.

Reconocer la importancia del contexto para la motivación humana no debería llevarnos empero hasta un excesivo abandono de nuestra concepción folk de la psicología humana. Tal y como argumenta Elster (2007), no se trata tampoco de abandonar la noción de individuo y de rendirnos ante un contexto todopoderoso. Se trataría más bien de descomponer la noción de carácter en una serie de tendencias de respuesta contingentes. En este sentido, deberíamos dejar de decir que una persona es honesta para pasar a utilizar formulaciones más complejas del estilo «es una persona que tiende a comportarse honestamente en situaciones del tipo X pero que, no obstante, puede resultar poco confiable en situaciones del tipo Y».

El diseño institucional y en particular el diseño de incentivos, constituye una herramienta poderosísima para definir las situaciones de un modo $u$

[13] Esto es lo que suele denominarse efectos deframing. VéaseKahneman y Tversky (2000).

Thémata. Revista de Filosofía №53 (2016) pp.: 107-118. 
otro y alterar así la motivación de los agentes. Una ley bien diseñada, por ejemplo, será aquella que, entre otras cosas, induzca a los agentes a categorizar las situaciones a las que se aplica como de un tipo tal que resulta pertinente cumplir con dicha ley y cooperar así en la promoción del bien público. En el otro extremo, una ley mal diseñada será aquella que desmotive a un agente que hasta ese momento estaba cumpliendo por voluntad propia motivado por una norma, por altruismo o similar ${ }^{14}$.

\section{Conclusiones}

El presente trabajo ha tratado dediscutir algunas vías y mecanismos a través de los cuales el diseño institucional podría contribuir a la generación y extensión social de la virtud cívica. Desde luego, el trabajo no ha pretendido dar por cerrada la discusión, sino simplemente exponer algunos elementos que bien podrían constituir la base para una exploración más sistemática del problema.

Quizá la principal conclusión que cabe extraer del ejercicio sea que, pese a la importancia de la cuestión, nuestros conocimientos sobre la misma son aún demasiado escasos y, desde luego, no permiten generar un catálogo de recomendaciones bien definidas sino, a lo sumo, algunas ideas generales. Por un lado, no sabemos prácticamente nada sobre cómo generar virtud cívica en aquellos contextos donde esta no existe. En lo que hace referencia a aquellos otros contextos en los que sí que existe una civilidad ampliamente difundida, pese a que sí que es posible identificar algunos mecanismos que podrían contribuir a cambiar la motivación de los villanos, no está claro de qué diseños institucionales concretos pueden contribuir a activarlos de forma óptima.

Probablemente, tal y como argumenta Pettit (1999) es a diseñar las instituciones de manera tal que aprovechen la virtud cívica existente en una determinada sociedad y que no la erosionen. Adicionalmente, tal y como argumentan Ayres y Braithwaite (1992), otro buen consejo para la creación de instituciones probablemente sea diseñarlas de manera que promuevan el cumplimiento y la cooperación social del máximo número posible de ciudadanos, independientemente de cuál sea su motivación, es decir, crear instituciones que economicen motivación.

[14] Nótese que, a la inversa, en una situación de cooperación entre villanos al regulador puede interesarle hacer todo lo contrario e introducir medidas que desactiven la cooperación.

Thémata. Revista de Filosofía №53 (2016) pp.: 107-118. 


\section{Bibliografia}

Ayres, I. y Braithwaite, J.: Responsiveregulation. New York: Oxford University Press, 1992.

Bicchieri, C.: The grammar of Society. The nature and dynamics of social norms.Cambridge: Cambridge University Press, 2006.

Elster, J.: Uvas amargas. Sobre la subversión de la racionalidad. Barcelona: Península, 1988.

Elster, J.: El cambio tecnológico. Investigaciones sobre la racionalidad y la transformación social. Barcelona: Gedisa, 1990.

Elster, J.: Psicología política. Barcelona: Gedisa, 1995.

Elster, J.: Alquimias de la mente. La racionalidad y las emociones. Barcelona: Paidós: El Roure, 2002.

Elster, J.: Explaining Social Behavior. More nuts and bolts for the social sciences.Cambridge: Cambridge UniversityPress, 2007.

Gambetta, D.: La Mafia siciliana: el negocio de la protección privada. México [etc.]: Fondo de Cultura Económica, 2007.

Hurka, T.: «Virtuousact, virtuousdispositions» en Análisis, 66 (1), 2006, pp. 69-76.

Kahneman, D. y Tversky, A. (eds.): Choices, values, and frames. New York: Cambridge University Press and Russell Sage Foundation, 2000.

Kuran, T.: Private truths, public lies: the social consequences of preference falsification. Cambridge, Mass.: Harvard University Press, 1995.

Kuran, T.: «Social mechanisms of dissonance reduction» enHedström, P. y Swedwerg, R.: Social Mechanisms. An analytical approach to social theory. Cambridge: Cambridge Press, 1998.

North, D.: Understanding the Process of Economic Change. Princeton: Princeton University Press, 2005.

Pettit, P.: Republicanismo. Una teoría sobre la libertad y el gobierno. Barcelona: Paidós, 1999.

Tena-Sánchez, J.: «Hacia una Definición de la Virtud Cívica» en Convergencia. Revista de Ciencias Sociales 53, 2010, pp. 311-337.

Tena-Sánchez, J.: «El Pluralismo Motivacional en la Especie Humana. Aportaciones Recientes de la Ciencia Social Experimental» en Papers. Revista de Sociologia 95(2), 2010b, pp. 421-439. 